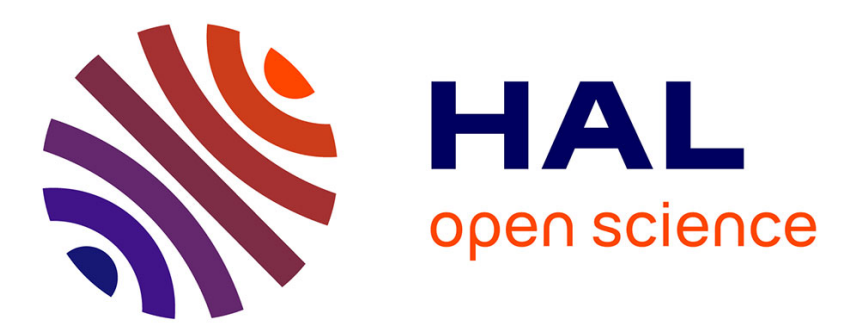

\title{
DEVELOPMENTS OF IRBIC AND QIRBIC IN DEFECT STUDIES : A REVIEW
}

\author{
A. Cavallini, A. Castaldini
}

\section{To cite this version:}

A. Cavallini, A. Castaldini. DEVELOPMENTS OF IRBIC AND QIRBIC IN DEFECT STUDIES: A REVIEW. Journal de Physique IV Proceedings, 1991, 01 (C6), pp.C6-89-C6-99. 10.1051/jp4:1991616 . jpa-00250701

\section{HAL Id: jpa-00250701 https://hal.science/jpa-00250701}

Submitted on 1 Jan 1991

HAL is a multi-disciplinary open access archive for the deposit and dissemination of scientific research documents, whether they are published or not. The documents may come from teaching and research institutions in France or abroad, or from public or private research centers.
L'archive ouverte pluridisciplinaire HAL, est destinée au dépôt et à la diffusion de documents scientifiques de niveau recherche, publiés ou non, émanant des établissements d'enseignement et de recherche français ou étrangers, des laboratoires publics ou privés. 


\title{
DEVELOPMENTS OF IRBIC AND QIRBIC IN DEFECT STUDIES: A REVIEW
}

\author{
A. CAVALLINI and A. CASTALDINI \\ Department of Physics, University of Bologna, Via Imerio 46, I-40126 Bologna, Italy
}

Abstract - Optical injection, as well as the electronic one, is of great use for semiconductor
material and component characterization, and in recent years several papers appeared dealing
with the analysis of the depth response of scanning light microscopy, the distribution of the
charge carriers generated by a focused light beam as well as the photoinduced current
dependence on the measurement configuration and the consequent imaging of defects in
semiconductors.

In these works a laser source is always used because of its features of monochromaticity, focusing and high intensity. Actually the light microscopy has become a widely accepted wafer mapping technique for investigating inhomogeneities and, besides, a method to evaluate diffusion length and surface recombination velocity of minority carriers.

We have applied the same principles of the scanning laser microscopy, but substituting filtered light coming from an halogen lamp to the laser source. This expedient, even though it involves some disadvantages about monochromaticity and focusing, allows, however, to easier handle light beam parameters as photon density and energy. We refer to this method as IRBIC (Infra-Red Beam Induced Current) since the photon energy of the scanning beam lies in the infrared portion of the spectrum. Object of our investigations is the current induced by the infrared light beam and, in more detail, the variations in defect imaging observed by changing penetration depth and intensity of the light. In addition, having a scanning light microscope at our disposal, we have modified the basic experimental setup so as to irradiate the back surface of the sample by monochromatic sub-band gap light. When the photon energy of the back surface irradiation coincides with the distance $E_{t}$ of a deep level within the forbidden gap from one of the allowed energy bands, a level-band transition occurs and, as a consequence, the concentration of the efficient recombination traps at the level $\mathrm{E}_{\mathrm{t}}$ changes. This process, involving the defect recombination activity, produces strong variations in the current induced by the beam scanning the opposite surface. The above said phenomenon, known for long time in bulk material examinations and named "photoconductivity quenching", has been applied by us to the scanning light microscopy and called "QIRBIC", that is "Quenched Infra-Red Beam Induced Current". Both the methods, IRBIC and QIRBIC, very sensitive to the occupancy factor of the defects, have been used to investigate the electrical activity of extended defects, namely dislocations, and their interaction with impurities and point defects. Besides, investigations have been performed on lamellar structure precipitates and on deformation-induced dislocation loops. 


\section{Introduction}

Charge Collection Scanning Microscopy (CCSM) has become an indispensable tool in the investigation of the physical properties of semiconducting materials and processed devices.

The potentiality of the CCSM is two-fold. First, it provides the possibility of determining such physical properties as minority carrier diffusion length and surface recombination velocity. Second, it is a powerful diagnostic method capable of detecting even subtle microstructural defects .

It is, thus, possible to connect materials microstructure, physical properties and, finally, device performance (for a wide range of literature see Holt /1/

It is, therefore, understandable the major role that the CCSM has assumed in the last few years.

In this framework there has been a resurgence in research on optical microscopy which the beam induced current method has been extended to.

It has, thus, been assessed the OBIC (Optical Beam Induced Current) method/2/ in which a light beam, instead of an electron beam, is used to excite electrons and holes in semiconductors. These are collected by an electric field built-in within the sample by a $p-n$ junction or a Schottky barrier, and the generated current can be measured.

By scanning the optical beam over the semiconductor an image is obtained where different recombination regions correspond to different grey densities.

The OBIC method uses a laser source (in the vast majority of the published works an He-Ne laser with a light wavelength equal to $6328 \AA$ ), in order to obtain an high signal/noise ratio.

Some years ago, we proposed a closely related approach /3/ to such optical imaging by substituting an halogen lamp to the laser. In this experimental arrangement interferential filters are used to make monochromatic the scanning beam light.

Since the light wavelength range used is the near infrared field, the method has been named IRBIC (Infrared Beam Induced Current). This optical arrangement has the major advantage that the light wavelength, and as a consequence the beam penetration depth, can easily be changed. Its disadvantage is the low intensity of the light source, which makes it necessary a high gain amplification of the output signal; on the other hand often this disadvantage turns into a useful characteristic of the technique when defects with very poor electrical activity are investigated $/ 4 /$.

The performances of the IRBIC setup have been extended by adding a second light source to the microscope in order of quenching the photocurrent induced by the beam.

This latter source is a globar whose light passes through a monochromator before impinging the sample. The light wavelength is modulated in such a range that its photon energy $h \nu$ is $h \nu<E_{g}$, where $E_{g}$ is the energy gap of the semiconductor under examination in order of giving rise to the optical freeing of the carriers at the defects.

Under these conditions the optical quenching of the photocurrent induced by the scanning beam occurs. In the usual definition, "quenching" is the effect by which a photocurrent and/or "dark" current through the specimen is reduced by illuminating the latter with another beam of light, and it is often referred to as "infrared quenching" of photoconductivity since it is the attenuation of short-wavelength excitation by the addition of long-wavelength excitation. Indeed, the photoquenching effect has been discovered early in the photoconduction investigations $/ 5,6 /$, but it has been thus far used as a bulk diagnostic method (see for example /7/). Instead, we combined the photoquenching process with the scanning beam induced current method: by this way the quenching radiation (hereafter called secondary or also background radiation) takes action on the magnitude of the scanning beam induced photocurrent (from now on named primary radiation).

As a consequence, the information achievable by means of the photoquenching, i,e. the position in the forbidden gap of the deep levels, can be ascribed exactly to the defect probed by the beam.

Thus we refer to this method as QIRBIC (Quenching Infrared Beam Induced Current). The present investigation deals with recent developments and results of the IRBIC and QIRBIC methods, performed with the intention of checking previous works, and of making a more thorough search of the power of the method. 


\section{Experimental layout}

Figure 1 shows the experimental arrangement used for both the IRBIC and QIRBIC investigations. The primary light, coming from an halogen lamp, passes through a pinhole to a microscope objective lens, so as the pinhole is imaged on the object.

Narrow band interferential filters can be put between lamp and pinhole so that the spot light impinging the sample is monochromatic; since the beam light wavelength ranges in the fundamental absorption region ( precisely from 505 to $853 \mathrm{~nm}$ ), the beam penetration depth d, defined as the reciprocal of the absorption coefficient $\alpha(\lambda)$, changes as a function of $\lambda$ (see Table $\mathrm{I}$ ).

\section{TABLE I}

\begin{tabular}{|l|c|c|c|c|c|c|c|}
\hline Wavelength (nm) & 505 & 550 & 633 & 671 & 707 & 768 & 853 \\
\hline Photon Energy (eV) & 2.45 & 2.25 & 1.95 & 1.84 & 1.75 & 1.61 & 1.45 \\
\hline Penetration Depth $(\mu \mathrm{m})$ & 0.66 & 1.40 & 2.62 & 3.69 & 4.64 & 8.54 & 15.92 \\
\hline
\end{tabular}

The specimen holder mechanically scans the object and constructs a raster image which is stored in a computer and then the frame is displayed as a video image.

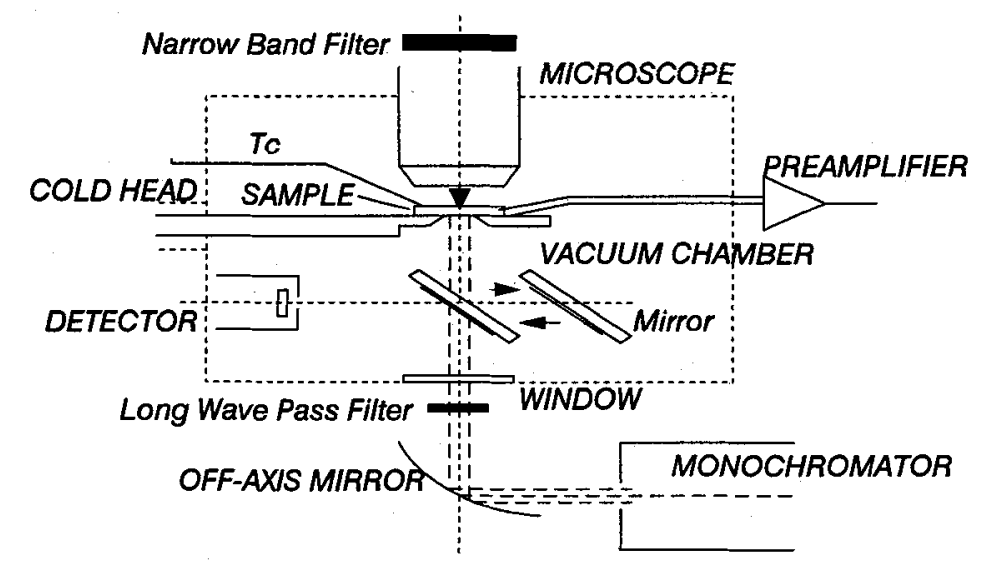

Figure 1 Layout for IRBIC and QIRBIC investigations.

The beam light is chopped so that it can be amplified by lock-in technique. The back surface of the sample is irradiated by long wavelength light coming out from a monochromator. It is filtered by longwave pass filters since only the first diffraction order is desired, while the other wavelengths which satisfy the grating equation need to be blocked.

A mirror may be interposed in the optical path for deflecting the secondary light to a photo-detector so as to measure the photons $\mathrm{cm}^{-2} \mathrm{~s}^{-1}$ incident on the back-surface

$$
\phi=\frac{\lambda I}{h c}=5.035 * 10^{12} \lambda I
$$

with $\lambda$ expressed in microns, I light intensity (in $\mu \mathrm{W} / \mathrm{cm}^{2}$ ), h Planck constant and $\mathrm{c}$ light velocity.

Both the primary and the secondary light injection levels can be adjusted so as to give the same incident power at each wavelength. In all the measurements performed low level injection regime has been used, that is the minority carrier concentration $\Delta n(\Delta p)$ is $\ll n_{0}$. A shielding ring prevents illumination of the 
electrical contacts. A cold stage has been provided to perform low temperature measurements, too.

\section{Results and Discussion}

We have performed both IRBIC and QIRBIC measurements to analyze first copper-contaminated floating zone silicon samples prepared by Gleichmann, Blumtritt and Heydenreich $18 /$, in which the minority charge collection is provided by a p-n junction. The starting sample, neutron doped, was diffused on the front side with $\mathrm{Ga}$, on the back with $\mathrm{P}$, obtaining junction depths of 30 and $60 \mu \mathrm{m}$, respectively. We examined the p-region where, besides dominant compact precipitates of copper silicide, also extended planar defects (lamellae) occur. These lamellar defects are usually limited by copper hexagonal precipitates and dislocations (Fig.2) and some of them present a bright EBIC contrast if they are reached by the primary electrons. The contrast $c$ is defined as:

$$
c=\frac{I^{\star}}{I_{0}}
$$

where: $I^{*}$ is the reduction of the IRBIC current at the defect, $I_{0}$ is the IRBIC signal level measured a large distance from the defect.

Figure 2 shows EBIC (a) and IRBIC (b,c,d,e) micrographs of two lamellar defects, one (A) presenting dark contrast and the other (B) bright contrast. Their depths have been localized at $3 \mu \mathrm{m}$ for the former and at $0.2-0.5 \mu \mathrm{m}$ for the latter $/ 8 \%$. The IRBIC images of both the lamellae have been obtained by changing the probing beam wavelength $\lambda=633 \mathrm{~nm}$ for the images (b) and (c) and $853 \mathrm{~nm}$ for (d)

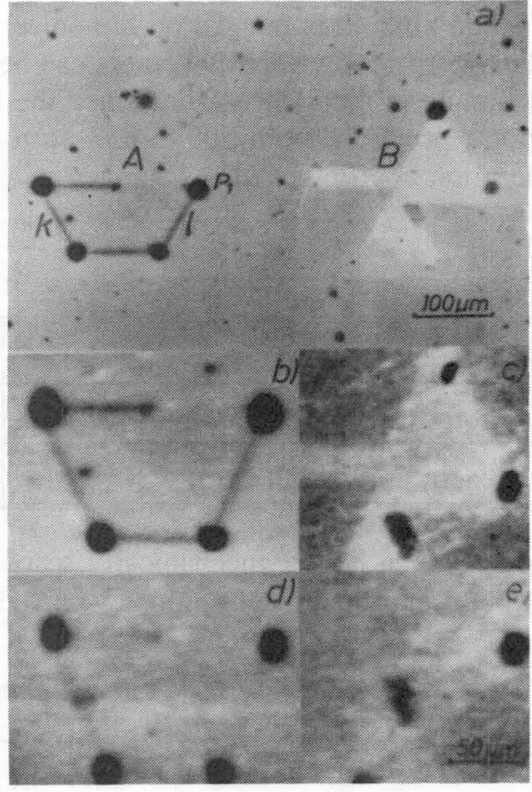

Figure 2. Micrographs of extended lamellar defects. EBIC image (a) [beam voltage $=17 \mathrm{kV}, \quad\left[\mathrm{b}=10^{-9} \mathrm{~A}\right]$ and IRBIC images: for $b$ and $c \lambda=633 \mathrm{~nm}$, for $d$ and $e$ $\lambda=853 \mathrm{~nm}$. and (e)) keeping constant the photon flux. From these pictures it appears clear that the defect imaging strongly depends on the beam wavelength. Therefore, the recombining behaviour of the different morphological types of defects (precipitates, dislocations and lamellae themselves) has been investigated as a function of the beam penetration depth as well as of the photon flux, or, in other words, of the electron-hole pair generation rate inside the generation volume. In this respect it is worth reminding that, in the focal region of a lens which focuses light at a distance $h_{0}$ into a material, the light distribution within the material is given by/9/:

$$
I(u, v)=\left.A^{2} \exp \left[-2 k \beta\left(h_{0}+z\right)\right] * \int_{0}^{1} J_{0}(v \rho) \cdot \exp \left(-0.5 j u \rho^{2}\right) * \exp \left[-0.5 \beta\left(u+u_{0}\right) \rho^{2}\right] \rho d \rho\right|^{2}
$$

where $\mathrm{J}_{0}$ is a zero order Bessel function of the first kind, A is a constant, $\rho$ a normalized variable in the plane of the aperture, $k \beta$ the amplitude absorption coefficient with $k$ wave number, $j$ the complex unity, $\mathrm{u}_{0}=\mathrm{kh}_{0} \sin ^{2} \alpha$, with $\sin \alpha$ numerical aperture, and $\mathrm{u}$ and $\mathrm{v}$ are optical coordinates given by:

$$
\begin{aligned}
& u=k z \sin ^{2} \alpha \\
& v=k r \sin \alpha
\end{aligned}
$$

Known the light intensity distribution, it is usual to define a generation volume within the $1 / \mathrm{e}$ contour for the analysis of the optical beam induced current imaging of defects $/ 2,10 \%$. However, in order to obtain a numerical estimate of the interaction of the light probe with the defects, this volume can be 


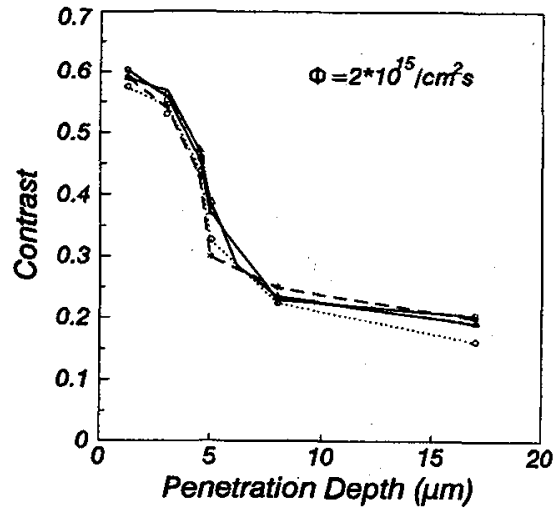

Figure 3. Contrast of the hexagonal precipitates of the lamella $A$ vs beam penetration depth.

crudely approximated $/ 11 /$ as a cone whose diameter $\mathrm{D}$ is given by the spot diameter plus two times the diffusion length $L$ and the height $H$ given by the penetration depth $d(\lambda)$ added to the diffusion length. In what follows this approximation will be used, assuming $\mathrm{L}=5 \mu \mathrm{m}$ for these specimens.

The contrast as a function of the beam penetration depth referring to the hexagonal precipitates - of Fig. 3 exhibits a trend rather similar to that observed in EBIC as a function of the primary electron range $R$ in ref. /8/: the beam induced current presents a sharp decrease for a penetration depth equal to $\sim 3 \mu \mathrm{m}$. However, substantial differences between the EBIC results and the IRBIC ones appear: the IRBIC maximum contrast is $60 \%$ whereas the EBIC one is $100 \%$, besides the IRBIC contrast decreases rapidly with $d$ showing a plateau when the penetration depth increases. This last finding refers to penetration depths exceeding those experienced by Gleichmann et al., who performed the EBIC investigation up to a primary electron range $R$ equal to $8.66 \mu \mathrm{m}$. The IRBIC results confirm, thus, the EBIC determination of the defect depth (about $3 \mu \mathrm{m}$ ) and the hypothesis of an highly recombining defect, rejecting at all that the precipitate plane acts as total diffusion barrier against the minorities carriers since even when the generation occurs prevalently between the surface and the defect a contrast of $60 \%$ is measured. A lower maximum value of $c$ in respect to the EBIC one can be attributed to the fact that, even at the shortest light wavelength used a portion of carriers, due to the injection way, is generated below the defect plane, that is between the defect and the junction. The other interesting feature relative to the shape of $c$ versus the penetration depth is the plateau corresponding to the longer wavelengths, not observed in EBIC observations. This behaviour of $c$, in good agreement with the theoretical prevision of Donolato $/ 12 /$, refers to penetration depths large enough so that the generation volume height $H$ becomes comparable to the junction depth.

IRBIC investigations of $c$ as a function of the photon flux $\phi$ have been performed referring to the precipitate $\mathrm{P} 1$ itself, the dislocations $\mathrm{k}$ and 1 and the bright contrast lamella of Fig.2.
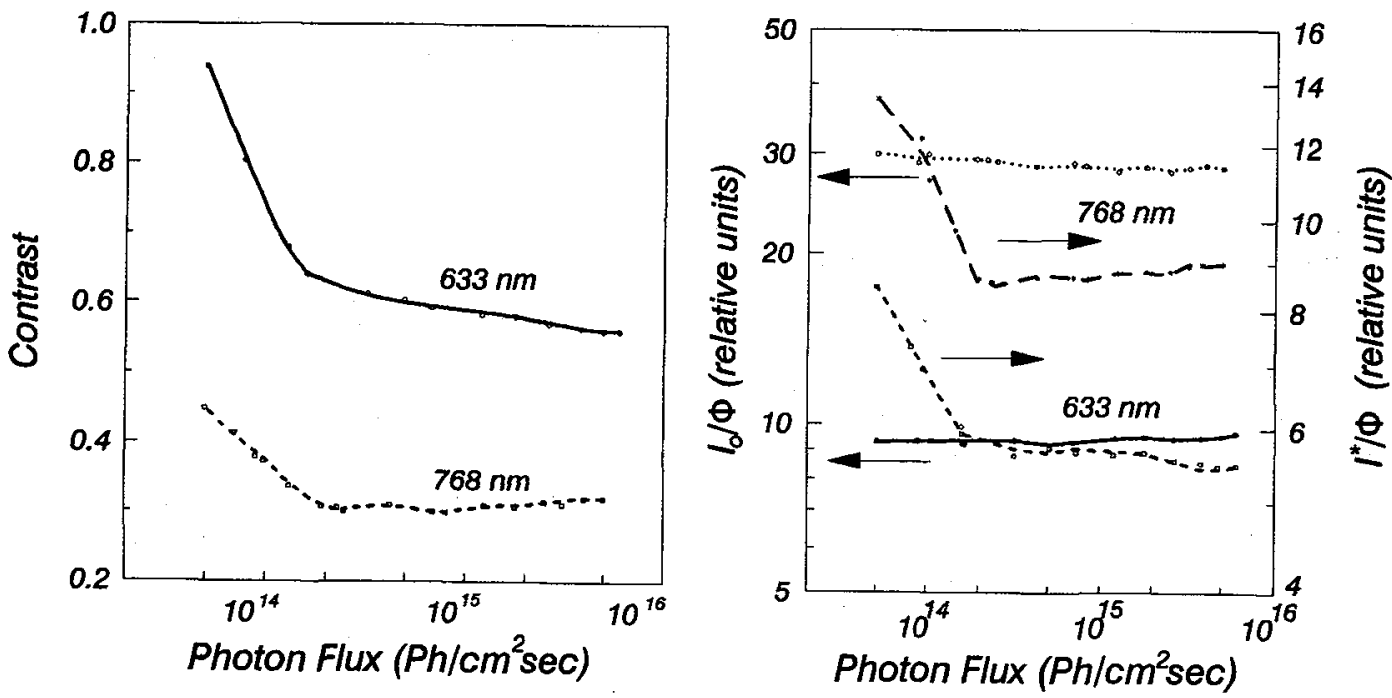

Figure 4a. Contrast of $P 1$ vs photon flux for probe $\lambda=633 \mathrm{~nm}$ and $768 \mathrm{~nm}$.

Figure $4 \mathrm{~b}$. IRBIC signal level $\mathrm{I}_{0}$ and reduction of the IRBIC current $\mathrm{I}^{*}$ at the precipitate, both normalized to the photon flux $\Phi$, vs $\Phi$. 
Figure $4 a$ shows the contrast relative to the precipitate $P 1$ for two beam light penetration lengths, $\lambda=633 \mathrm{~nm}$ corresponding to a generation volume $\mathrm{V}$ prevalently located between the sample surface and the defect, and $\lambda=768 \mathrm{~nm}$ for which the larger portion of $V$ is between the defect and the junction plane.

The two curves have the same trend, but the $633 \mathrm{~nm}$ one presents a larger contrast. The trend observed is rather unusual: at low photon fluxes the contrast is much sensitive to changes in injection levels and decreases when the injection level increases (regime I), then a plateau follows at further increases of $\phi$ (regime II). In order to observe in greater details the electrical activity changes of the precipitate, in Fig.4b the signal current $I_{0}$ and the defect current reduction $I^{*}$ were both normalized to the photon flux $\phi$ and plotted versus the $\ln (\phi)$, following the procedure of Norman and Holt/13/. While the signal current $\mathrm{I}_{0}$ is directly proportional to $\phi$ in the whole injection range explored, the behaviour of $\mathrm{I}^{*}$ mirrors that of $c$, with a first stage of linear dependence on $\ln (\phi)$, sharply becoming independent of the injection level at the same value of $\phi$ for both the penetration depths. The regime $I$ has been predicted by the Wilshaw-Booker (W-B)/14/ theory and confirmed by Norman et al./13/ but, to the knowledge of the authors, the regime II has never been observed. In order to compare the present results with those obtained by EBIC, the total generation rate $\mathrm{G}$, given by/15/:

$$
G=1.6 \cdot 10^{18} I_{\text {beam }} E_{\text {beam }}
$$

where $I_{\text {beam }}$ is in amps and $E_{\text {beam }}$ in volts, has been calculated for $E_{\text {beam }}=15 \mathrm{kV}$ and divided by the generation volume (in the uniform sphere generation approximation) so as to obtain the generation rate of hole-electrons pairs per unit volume $\mathrm{g}$. The optical counterpart of $\mathrm{G}$ can be written as /16/

$$
G=\frac{P_{b} Q}{q E_{g}}(1-\gamma)
$$

with $P_{b}$ beam power, given by the irradiance $I$ (see eq.1) times the area of the spot, $Q$ the quantum efficiency for carrier generation $/ 17 /, \gamma$ the reflectivity coefficient, $q$ the electronic charge and $E_{g}$ the band gap. This expression, divided by the optical generation volume (in the cone approximation) yields the equivalent optical $g$. In Table II values of the above parameters are reported for comparison.

Table II

\begin{tabular}{|c|c|c|c|c|c|c||}
\hline $\begin{array}{c}\mathrm{Ib} \\
(\mathrm{A})\end{array}$ & $\begin{array}{c}\mathrm{G} \\
(\mathrm{pairs} / \mathrm{s})\end{array}$ & $\begin{array}{c}\mathrm{g} \\
\left(\text { pairs } / \mathrm{cm}^{3} \mathrm{~s}\right)\end{array}$ & $\begin{array}{c}\phi \\
\left(\mathrm{cm}^{-2} \mathrm{~s}-1\right)\end{array}$ & $\begin{array}{c}\mathrm{P}_{\mathrm{b}} \\
(\mathrm{W})\end{array}$ & $\begin{array}{c}\mathrm{g}_{633} \\
\left(\text { pairs } / \mathrm{cm}^{3} \mathrm{~s}\right)\end{array}$ & $\begin{array}{c}\mathrm{g}_{768} \\
(\text { pairs/cm }\end{array}$ \\
\hline $1 \cdot 10^{-12}$ & $2.4 \cdot 10^{7}$ & $6.1 \cdot 10^{18}$ & $1.0 \cdot 10^{13}$ & $3.5 \cdot 10^{-14}$ & $4.4 \cdot 10^{14}$ & $2.5 \cdot 10^{14}$ \\
\hline $1 \cdot 10^{-11}$ & $2.4 \cdot 10^{8}$ & $6.1 \cdot 10^{19}$ & $1.0 \cdot 10^{14}$ & $3.9 \cdot 10^{-13}$ & $4.9 \cdot 10^{15}$ & $2.8 \cdot 10^{15}$ \\
\hline $1 \cdot 10^{-10}$ & $2.4 \cdot 10^{9}$ & $6.1 \cdot 10^{20}$ & $1.0 \cdot 10^{15}$ & $3.6 \cdot 10^{-12}$ & $4.5 \cdot 10^{16}$ & $2.6 \cdot 10^{16}$ \\
\hline
\end{tabular}

As appears in Table II, the injection level relative to the present results is very low. Therefore it could seem surprising, on the basis of the W-B theory, that the defect examined is not approaching the equilibrium charge. However, analogous finding relative to dislocations in $\mathrm{GaAs} / 18 /$ has been found, and tentatively attributed to the higher concentration of dislocation levels than are associated with dislocations in $\mathrm{Si}$. The same justification can be reported in the present case bearing in mind that the defects examined are $\mathrm{Cu}$ decorated dislocations or even precipitates, like the case at issue. Similar behaviour has been observed by IRBIC investigations of the dislocations $\mathrm{k}$ and 1 for $\lambda=633 \mathrm{~nm}$, even if the onset of the dependence on $\phi$ shifts towards highest values, while for $\lambda=768 \mathrm{~nm}$ the contrast $c$ is constant $(c=4-5 \%)$. Since the recombination efficiency depends on the line charge, these findings could be attributed to a lower defect level concentration than that presented by the precipitate, giving rise to the predicted regime $I$ in the first portion of the diagram relative to the shorter penetration depth and to the regime of constant $c$ (corresponding to a dislocation charge close to the equilibrium) for the longer 
$\lambda$, at which the defect experiences a higher generated carrier concentration.
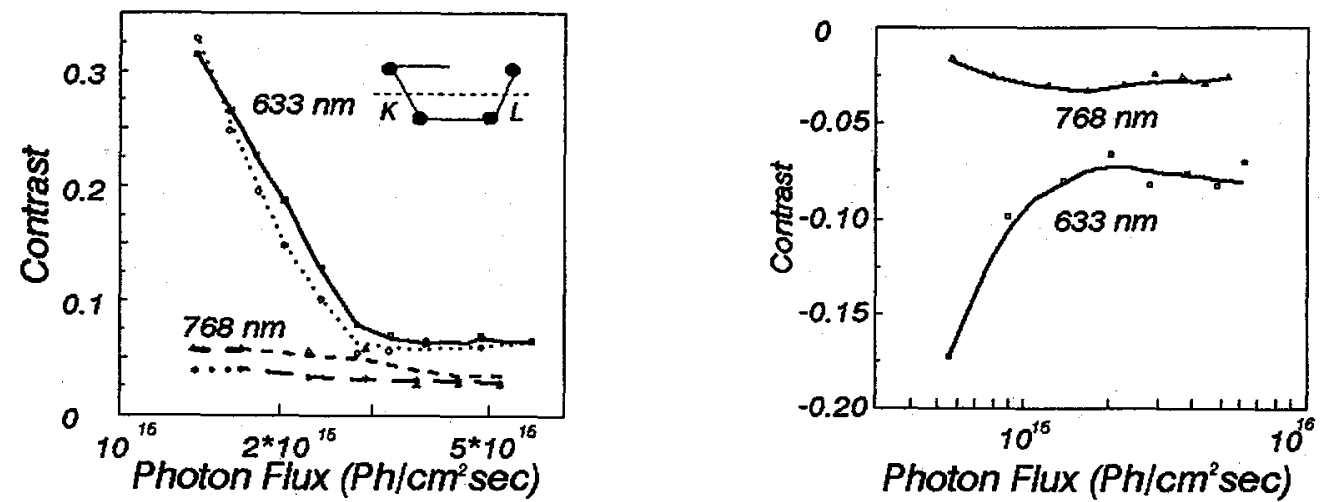

Figure 5. Contrast $\mathrm{c}$ of the dislocations $\mathrm{k}$ and 1 vs $\Phi$ for probe light wavelength equal to $633 \mathrm{~nm}$ and $768 \mathrm{~nm}$.

Figure 6. Contrast $c$ of the lamella $B$ vs $\Phi$ for $\lambda=633 \mathrm{~nm}$ and $768 \mathrm{~nm}$.

The last defect investigated is the lamella B of Fig.2, whose contrast vs $\phi$ is reported (Fig. 6).

It was already demonstrated/19/ that the bright contrast arises from a shielding effect of the minority carriers against the surface recombination by the negatively charged defect plane, provided the majority of carriers is generated below the defect plane. The IRBIC measurements at $633 \mathrm{~nm}$ takes into evidence the progressive reduction of the surface effect (the density of the excess carriers is controlled by bulk and surface recombination) since the negative contrast increases approaching zero (see also Fig.2e) by increasing $\phi$, that is a progressively smaller proportion of the beam induced carriers recombine at the surface. For $\lambda=768 \mathrm{~nm}$ the portion of carriers generated between surface and lamella is a very small fraction of those generated, and thus the contrast does not experience the effect of the surface by changing the photon flux.

The same defects have been investigated by the QIRBIC method. Figure 7 refers to the QIRBIC contrast of the precipitate $\mathrm{P} 1$ : this defect has been thought a reliable test for QIRBIC search because of its known origin (CuSi precipitates) determined by an energy dispersive X-ray analysis/8/.

As told before, by the QIRBIC method contrast profiles can be obtained which are associated with changes of the occupancy factor $f$ of the energy levels associated to the dislocation lying in the forbidden gap. Thus it has to be feasible to observe contrast changes corresponding to transitions between the defect levels and one of the allowed bands mirroring the presence of copper. Usually, when quenching effects involving multi-level defects are present, the photocurrent response pattern gets very complex. However, as refers to the above mentioned precipitates, the contrast findings are simplified by the presence of the very high concentration of copper. This bears out by the QIRBIC profile at room temperature, where three well defined minima appear at $0.24 \mathrm{eV}, 0.39 \mathrm{eV}$ and $0.53-0.54 \mathrm{eV}$, corresponding $/ 20$ / to the deep levels introduced in the forbidden gap by $\mathrm{Cu}$. At lower temperature the contrast profile shifts downwards /21/ and its features get more complexes: the interpretation of

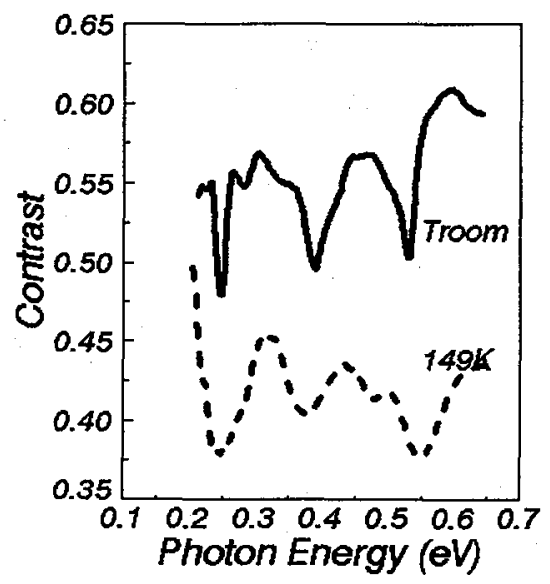

Figure 7. QIRBIC contrast vs secondary illumination photon energy at the temperature $\mathrm{T}=300 \mathrm{~K} \quad(-)$ and $T=149 \mathrm{~K}(.$.$) . Scanning beam$ wavelength $\lambda=633 \mathrm{~nm}$. 
these data is not a simple one, but one reason could stem from the presence at the defect of multiple energy levels becoming active lowering the temperature.

In order of checking once and for all the reliability of the QIRBIC method, slip lines on purpose gold decorated have been investigated. For this aim n-type silicon, $P$ doped $(\rho=1002 \mathrm{~cm})$, has been gold evaporated and subsequently processed $/ 22 /$ so as to decorated dislocations previously introduced. Photoconductivity normalized to incident power as been measured as a function of the light (Fig.8a) and the spectrum obtained compared to that reported in ref./23/. Since our findings are in good agreement with those reported in literature, a search has been made by QIRBIC for the acceptor level at $(0.54 \pm 0.02) \mathrm{eV}$ from the conduction band reported in $/ 23 /$. The QIRBIC investigation (Fig.8b) presents a sharp minimum precisely at $0.55 \mathrm{eV}$.
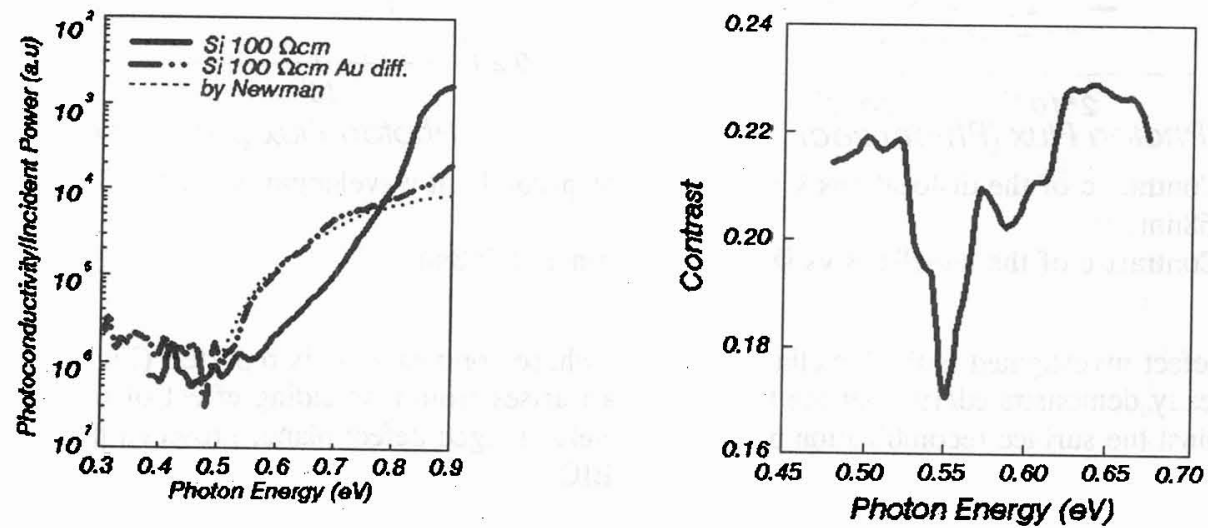

Figure 8a. Photoconductivity spectra of as-grown and Au diffused Si. Data from ref.23 are also reported.

Figure 8b. QIRBIC contrast of gold decorated dislocation. Scanning beam $\lambda=633 \mathrm{~nm}$.

Corroborated by the relationship between QIRBIC contrast profile features and deep level position in the forbidden gap by this test, both IRBIC and QIRBIC methods have been used to examine 30/30 and 30/90
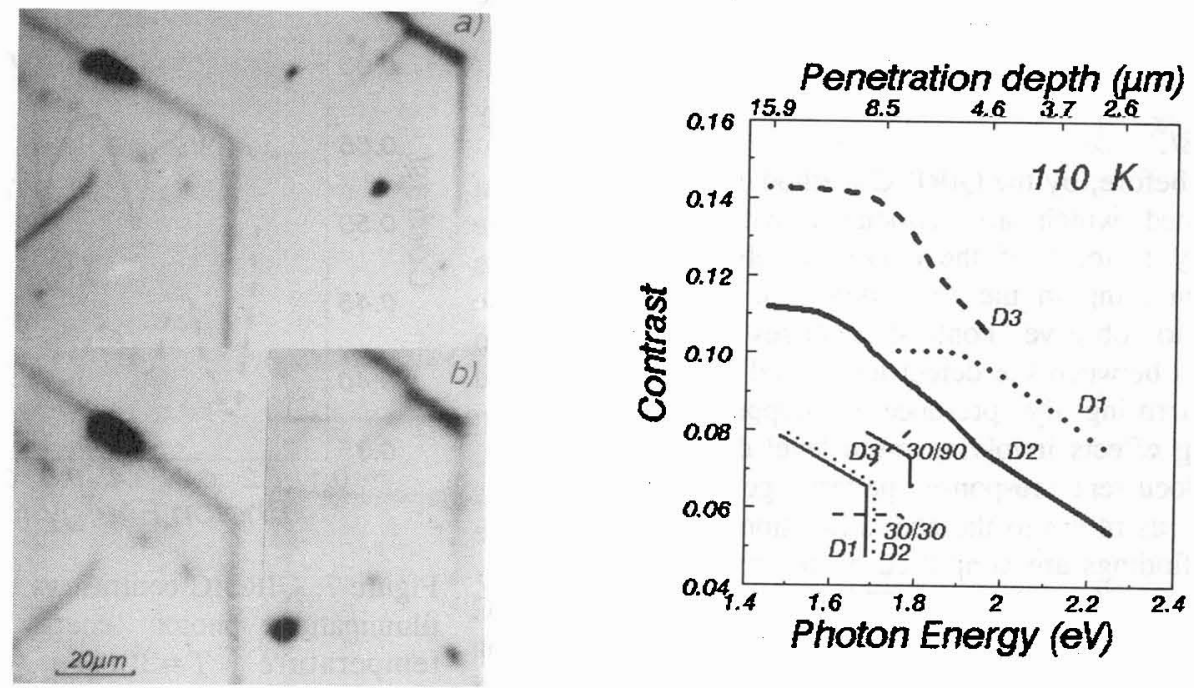

Figure 9a. EBIC (a) (Ebeam =20KV) and IRBIC (b) $(\lambda=550 \mathrm{~nm})$ micrographs of dislocation loops. Figure $9 \mathrm{~b}$. IRBIC contrast of dislocation imaged in Fig.9a at temperature $\mathrm{T}=110 \mathrm{~K}$. 
dislocations (the specimens used for this work were provided by Professor $\mathbf{H}$. Alexander and colleagues, Cologne University, FRG.). The results here reported refer to a Si specimen $\mathrm{P}$ doped, doping concentration $7 * 10^{15} \mathrm{~cm}^{-3}$, where dislocation loops have been produced (EBIC micrograph in Fig.9a). The collecting Schottky barrier has been obtained by a gold evaporation. Figure $9 \mathrm{~b}$ shows the IRBIC contrast profiles of the dislocations imaged in Fig.9a (sketch on the left bottom) against the beam penetration depth $\mathrm{d}$ at temperature $\mathrm{T}=110 \mathrm{~K}$ and with a photon flux $\phi=2 * 10^{15} \mathrm{~cm}^{-2} \mathrm{~s}^{-1}$. The contrast trend of all the dislocations is as expected on the basis of the beam induced current theory $/ 12 \%$. Nevertheless, even if a complete detailed comparison will require a thorough search, it is worth noting that the dislocation D2 is not visible in the EBIC micrograph, while it presents a contrast $\geq 5 \%$ in the IRBIC measurements.

QIRBIC investigations of D1 and D2 (Fig.10), obtained with scanning probe light $\lambda=633 \mathrm{~nm}$ and $\phi=2 * 10^{15} \mathrm{~cm}^{-2} \mathrm{~s}^{-1}$ on the front side of the sample, take into evidence another interesting difference between these dislocations: though on average the QIRBIC contrasts are comparable, the two curves are slightly, but significantly, different. In particular the minimum at $0.72 \mathrm{eV}$ is much more relevant for the dislocation D2.

In order to gain an insight into the QIRBIC contrast findings, the following experiment has been performed. The dislocations D1 and D3, both presenting a minimum at $0.40 \mathrm{eV}$ and $0.72 \mathrm{eV}$, have been first of all imaged in IRBIC mode (experimental conditions reported in Fig. 11), then a Si filter has been interposed between the scanning beam and the sample in order to observe the photoconductivity response due to the fundamental absorption edge only, so as to reduce as much as possible the intrinsic photoconductivity. In this case an increase of the beam induced current occurs at

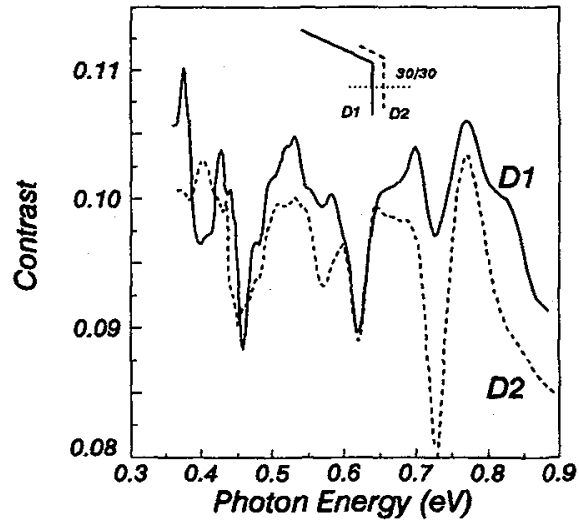

Figure 10 QIRBIC contrast vs quenching photon energy of the dislocation D1 and D2 at room temperature ( scanning probe wavelength $\lambda=633 \mathrm{~nm}$ ). the dislocations. Then the secondary light source has been turned on with such a wavelength that the photon energy $h \nu=0.40 \mathrm{eV}$ : a current increase occurs again, but the contrast is slightly different at D1 and D3, that is $c=-28 \%$ and $c=-35 \%$, respectively. If the secondary light photon energy is, instead, $h \nu=0.72 \mathrm{eV}$ a positive contrast $\mathrm{c}=28 \%$ is observed. A satisfactory explanation of all the effects reported above, particularly those related to the numerical value of the quenching induced contrast, has not been forthcoming. However, it is felt that for heuristic purposes tentative hypotheses may be of value. The optical properties of a given defect are totally defined by the optical cross-sections for electrons and holes $\sigma_{\mathrm{n}, \mathrm{o}}$ and $\sigma_{\mathrm{p}, \mathrm{o}}$, which are functions of photon energy. Therefore, from the principle of detailed balance holding for radiative conditions, the rate equation governing the occupation of a defect state of total concentration $\mathrm{N}_{\mathrm{T}}$ are/24/

$$
\frac{d N}{d t}=\left(c_{n}+e_{p}+e_{p, o}\right)\left(N_{T}-N\right)-\left(c_{p}+e_{n}+e_{n, 0}\right) N
$$

with $\mathbf{N}$ concentration of defects occupied by electrons, $c_{n}$ and $c_{p}$ electron and hole capture cross-sections, $e_{\mathrm{n}}$ and $e_{\mathrm{p}}$ emission rates and $e_{\mathrm{n}, \mathrm{o}}$ and $\mathrm{e}_{\mathrm{p}, \mathrm{o}}$ optical emission rates, that is the rates to optically remove carriers from the defect state. The former term expresses the recombination rate, the latter the generation rate; thus we will let

$$
\frac{d N}{d t}=R-G
$$

As known, light can produce both excitation of photoconductivity, exciting from levels to the conduction band, as occurs in our experiment when light corresponding to the fundamental edge impinges the defect, and quenching of photoconductivity, exciting from the valence band to the sensitizing centers. When the 
secondary light is turned on with $h \nu=\left(\mathrm{E}_{\mathrm{c}}-\mathrm{E}_{\mathrm{v}}\right)$, it is possible to promote trapped electrons from the defect to the conduction band by photoelectric effect, which results in an increase of the photoconductivity.

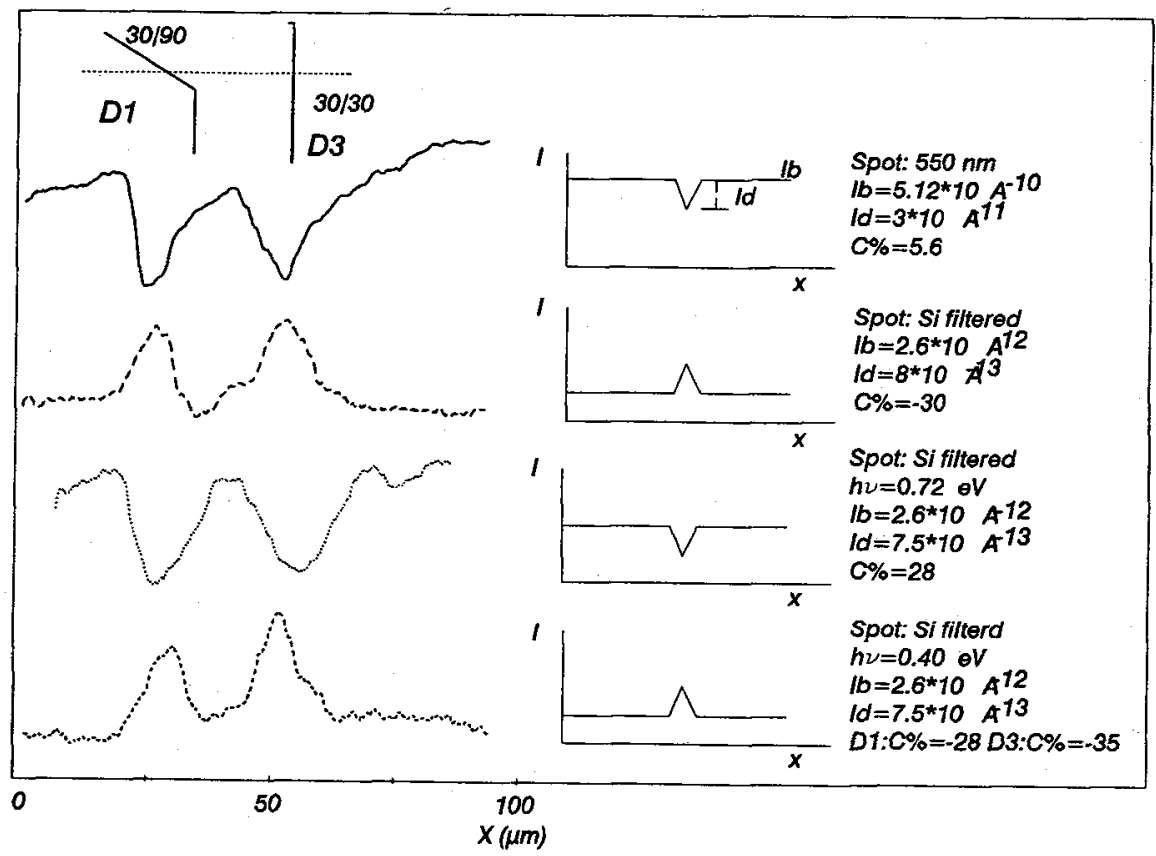

Figure 11 On the left beam induced current profiles of D1 and D3 ( scanning direction sketched on the top), on the right irradiation conditions

The output information is complicated by the fact that when $h \nu$ is still increased, it becomes feasible to repopulate the recombination centers optically via transitions from the valence band to the center. The probability of these last transitions will be enhanced when $h \nu \geq\left(\mathrm{E}_{\mathrm{l}}-\mathrm{E}_{\mathrm{v}}\right)$. In this case two competing processes, the defect level filling and emptying, occur and control the population of the center, prevailing one on the other depending on the relative optical emission rates. In the investigated case at $h \nu=0.72$ the recombination $R$ prevails on the generation $\mathbf{G}$. Therefore, even if very complex spectra result when multi-level defects are involved, this procedure is able to distinguish whether a QIRBIC minimum corresponds to level-conduction band edge or valence band-level transitions.

\section{Conclusions}

In the above analyses the IRBIC and QIRBIC method potentials to investigate the defect recombination behaviour have been explored. It has been shown that IRBIC measurements can give much information to identify the nature of both "strong" (precipitates) and very "weak" defects (dislocations not imaged in EBIC), where strong and weak are relative to the defect recombination efficiency, being able to change in a wide range the experimental parameters (light intensity distribution and carrier generation). Besides, the very low generation rate often turns into a powerful tool of investigation.

The problem of recombination still retains a large measure of complexity, first of all allowing for multilevel defects. However the QIRBIC method, even if a complete detailed definition of its potentials needs to receive a great deal of attention, has been demonstrated to be a straightforward way for detailed studies of the spatial distribution of deep level defects. 
Acknowledgements

The authors wish to thank $\mathrm{H}$. Alexander and $\mathrm{R}$. Gleichmann and colleagues for kindly supplying the samples examined and information, and the INFM for support.

\section{REFERENCES}

/1/ HOLT,D.B and JOY,D.C (eds.), Sem Microcharacterization of Semiconductors, Academic Press London (1989)

12/ WILSON,T and McCABE,E.M, Optik 75 (1986) 11

13/ CAVALLINI,A, GONDI,P and CASTALDINI,A. Izv.Akad.Nauk. (USSR)(1986) 223.

14/ CASTAldini,A, CAVAllini,A, POGGI,A, and SUSI,E. Appl.Phys.A $\underline{48}$ (1989) 431.

15/ BUBE,R.H, Phys. Rev. 99 (1955) 1105

16/ ROSE,A, Phys.Rev. 97 (1955) 322

/7/ YAKIMOV,E.B, EREMENKO,V.G and NIKITENKO,V.I, Sov.Phys.Semicond. 10 (1976) 231

18/ GLEICHMANN,R, BLUMTRITT,H and HEYDENREICH,J, Phys.stat.sol.(a) $\underline{78}$ (1983) 527

19/. BORN,M and WOLF,E, Principles of Optics, Pergamon Press, Oxford, (1987).

/10/ WILSON,T and McCABE,E.M, J.Appl.Phys. 59 (1986) 2638

/11/ WILSON,T. and SHEPPARD,C. Scanning Optical Microscopy. Academic, London (1984) 179.

/12/ DONOLATO,C, Phys.Stat.Sol. (a) 66 (1981) 445.

/13/ NORMAN,C,E. and HOLT,D,B. in Microsc.Semicond.Mater. Oxford (1991) in press.

/14/ WILSHAW,P,R. and BOOKER,G,R. Microsc.Semicond.Mater. Oxford 76 (1985) 329.

/15/ BERZ,F and KUIKEN,K, Solid.State Electron. 19 (1976) 437

/16/ DAVIDSON,S.M and DIMITRIADIS,C.A, J.of Microscopy $\underline{118}$ (1980) 275

/17/ DAVIDSON,S,M. INNES,R,M. LINDSAY,S,M. Solid.State.Electron.25 (1982) 261 .

/18/ WILSHAW,P,R. FELL,T,S. and BOOKER,G,R. in Point and Extended Defects in Semiconductors, Benedek,G, Cavallini,A, Schröter eds. NATO ASI Series B202 (1989) 243.

/19/ BLUMTRITT,H, KITTLER M and SEIFERT W, Inst.Phys.Conf.Ser. 104 (1989) 233

120/ SZE,S,M. Physics of Semiconductor Devices. John Wiley \& Sons NY (1981)

121/ CASTALDINI,A.,CAVALLINI,A and CAVALCOLI,D. Inst.Phys.Conf.Ser. 104 (1989) 169.

/22/ RADZIMSKI,Z.J,ZHOU,T.Q, BUCZKOWSKI,A, ROZGONYI G.A, FINN,D, HELLWIG,L. ROSS,J. Appl.Phys.Lett. in press.

/23/ COLLINS,C.B, CARLSON,R.O and GALLAGHER,C.J, Phys.Rev. 105 (1957) 1168

/24/ MILLER,G,L. LANG,D,V. and KIMERLING,L,C. Ann.Rev.Mater.Sci. (1977) 377. 